\title{
Fusarium fujikuroi Species Complex Associated With Rice, Maize, and Soybean From Jiangsu Province, China: Phylogenetic, Pathogenic, and Toxigenic Analysis
}

\author{
Jianbo Qiu, ${ }^{1}$ Yunan Lu, ${ }^{2}$ Dan He, ${ }^{1}$ Yin-Won Lee, ${ }^{3}$ Fang Ji, ${ }^{1,4}$ Jianhong Xu,,${ }^{1,5,}$ and Jianrong Shi ${ }^{1,5,+}$ \\ ${ }^{1}$ Jiangsu Key Laboratory for Food Quality and Safety - State Key Laboratory Cultivation Base, Ministry of Science and Tech- \\ nology; Key Laboratory for Control Technology and Standard for Agro-product Safety and Quality, Ministry of Agriculture and \\ Rural Affairs; Collaborative Innovation Center for Modern Grain Circulation and Safety; and Institute of Food Safety and Nu- \\ trition, Jiangsu Academy of Agricultural Sciences, Nanjing 210014, China \\ ${ }^{2}$ College of Marine Life and Fisheries, Jiangsu Ocean University, Lianyungang 222005, China \\ ${ }^{3}$ Department of Agricultural Biotechnology, Seoul National University, Seoul 08826, Republic of Korea \\ ${ }^{4}$ Microbiology Discipline, School of Life Sciences, University of KwaZulu-Natal, Westville Campus, Durban, South Africa \\ ${ }^{5}$ School of Food and Biological Engineering, Jiangsu University, Zhenjiang 212013, China
}

\begin{abstract}
Species belonging to the Fusarium fujikuroi species complex (FFSC) are of vital importance and are a major concern for food quantity and quality worldwide, as they not only cause serious diseases in crops but also produce various mycotoxins. To characterize the population structure and evaluate the risk of poisonous secondary metabolites, a total of 237 candidate strains were isolated from rice, maize, and soybean samples in Jiangsu Province, China. Species identification of the individual strain was accomplished by sequencing the translation elongation factor $1 \alpha$ gene $(T E F-1 \alpha)$ and the fumonisin (FB) synthetic gene (FUM1). The distribution of Fusarium species among the different crops was observed. The maize seeds were dominated by $F$. proliferatum (teleomorph, Gibberella intermedia) and $F$. verticillioides (teleomorph, G. moniliformis), whereas F. fujikuroi (teleomorph, G. fujikuroi) was the most frequently

species pathogenicity and toxigenicity were studied. All of the species reduced the rice seed germination rate, with no significant differences. $F$. fujikuroi showed two distinct patterns of influencing the length of rice seedlings, which were correlated with FBs and gibberellic acid synthesis. FBs were mainly produced by $F$. verticillioides and $F$. proliferatum. $F$. proliferatum and $F$. fujikuroi also produced moniliformin and beauvericin. The toxigenicity of $F$. andiyazi (teleomorph, G. andiyazi) was extremely low. Further analysis indicated that the sequence variations in $T E F-1 \alpha$ and the differences in the expression levels of the toxin synthesis genes were associated with the diversity of secondary metabolites in $F$. fujikuroi strains. These findings provide insight into the populationlevel characterization of the FFSC and might be helpful in the development of strategies for the management of diseases and mycotoxins.
\end{abstract} isolated species from rice and soybean samples. In addition, phylogenetic analyses of these strains were performed, and the results suggested clear groups showing no obvious relationship with the origin source. FFSC
Keywords: cereal, Gibberella fujikuroi species complex, pathogenicity, phylogence, toxigenicity
Agricultural crops are not only crucial nutrient-rich food sources for human survival, but they are also important industrial and agricultural feed raw materials. Consequently, the quality and safety of agricultural crops and their products is of great significance to human and animal health. As a result of the influence of the agricultural environment and inputs, there are various food security issues associated with agricultural products, such as the presence of heavy metals and pesticide residues (Clemens and Ma 2016; Grewal et al. 2017). According to previous studies, mycotoxins have gradually become the leading threat, especially Fusarium toxins produced by Fusarium species under favorable environmental conditions (Lee and Ryu 2017).

${ }^{\dagger}$ Corresponding authors: J. H. Xu; xujianhongnj@126.com, and J. R. Shi; shiji@jaas.ac.cn

Funding: This work was supported by grants from the National Natural Science Foundation of China (31701748 and 31772118), Jiangsu Agriculture Science and Technology Innovation Fund (CX(17)1003), International Science \& Technology Cooperation Program of China (2016YFE0112900), Shanghai Agriculture Applied Technology Development Program, China (2019-02-08-00-12F01148), and National Key R\&D Program of China (2018YFD0200500).

*The $\boldsymbol{e}$-Xtra logo stands for "electronic extra" and indicates that one supplementary figure and one supplementary table are published online.

The author(s) declare no conflict of interest.

Accepted for publication 20 February 2020.

(c) 2020 The American Phytopathological Society
The $F$. fujikuroi species complex (FFSC) is an important component of the genus Fusarium and consists of more than 50 phylogenetically distinct species (O'Donnell et al. 2015). Among the members of the FFSC, $F$. verticillioides (teleomorph, Gibberella moniliformis), F. fujikuroi (teleomorph, G. fujikuroi), and F. proliferatum (teleomorph, $G$. intermedia) are well known for their abilities to cause devastating cereal diseases, such as rice bakanae, maize ear rot, and soybean root rot, leading to considerable reductions in crop yields and economic income.

Another significant challenge is that members of the FFSC can produce a variety of mycotoxins, including fumonisins (FBs) (Rheeder et al. 2002), fusaric acid (Bacon et al. 1996), moniliformin (MON) (Marasas et al. 1986), gibberellic acids (GAs) (Cerdá-Olmedo et al. 1994), beauvericin (BEA) (Logrieco et al. 1998), fusaproliferin (FUP) (Moretti et al. 1996), and enniatins (Nicholson et al. 2004), that contaminate food and cause chronic and acute toxicity to humans and livestock. Among these metabolites, FBs are the most frequently detected, with relatively high concentrations, especially in rice and maize (Ferrigo et al. 2016). Many studies have suggested that FBs inhibit sphingosine- $N$-acyltransferases, which are involved in sphingolipid biosynthesis (Abado-Becognee et al. 1998). Although there is currently no direct evidence that FBs are associated with serious human and animal health problems, the high occurrence of human esophageal cancer is apparently related to the consumption of maize-based products contaminated with FBs (Gelderblom et al. 2001). In addition, FBs exhibit certain degrees of neurotoxicity, cytotoxicity, immunotoxicity, and hepatotoxicity (Stockmann-Juvala and Savolainen 2008).

However, there are significant differences in the levels and types of mycotoxins produced by individual species. $F$. verticillioides and $F$. proliferatum are major FB producers, whereas other species 
in the FFSC mainly produce certain amounts of BEA and FUP (Leslie et al. 2004). GA is usually synthesized by $F$. fujikuroi strains. In research studies, two groups of $F$. fujikuroi were distinguished based on the production of GAs and FBs (Niehaus et al. 2017; Suga et al. 2019). These toxic metabolites have been suggested as important tools for species discrimination in the FFSC (Leslie 1995; Moretti et al. 1996)

Some FFSC strains are similar in colony morphology and biology, which makes it difficult to directly differentiate strains. Phylogenetic analysis based on DNA sequence diversity plays a crucial role, and many molecular markers, such as internal transcribed spacer regions, $\beta$-tubulin, MAT alleles, and calmodulin are currently used; however, $\mathrm{H} 3$ histones and translation elongation factor $(T E F-1 \alpha)$ sequences appear to be the most useful in taxonomic studies of fungi, especially for the FFSC as well as other members of the Fusarium genus (O'Donnell et al. 2000; Proctor et al. 2013). The latest findings suggested that genes and other sequences involved in the mycotoxin biosynthesis pathway could be good targets in phylogenetic studies, as they have the advantage of possible use in combined approaches for the diagnostics of mycotoxin production abilities (Stępien 2014). Genes from the FUM cluster, responsible for the FB biosynthetic pathway, have been successfully utilized as successful markers in phylogenetic research on toxin-producing Fusarium species (Baird et al. 2008; Proctor et al. 2013).

In our previous study, we explicitly demonstrated that $F$. verticillioides and $F$. proliferatum were prevalent in maize samples from eastern China (Qiu et al. 2015). Nevertheless, we had little information about toxin production in these species and Fusarium occurrence in other crops. The main aims of this study were to determine the FFSC composition in different hosts by analyzing the differences in the sequences of $T E F-1 \alpha$ and $F U M 1$, a polyketide synthase gene required for FB biosynthesis (Proctor et al. 1999). Moreover, the ability of the identified strains to produce mycotoxins and cause diseases was evaluated. The results will contribute to the development of prevention and control strategies for Fusarium species and their metabolites in agricultural crops.

Table 1. List of isolates analyzed in this study

\begin{tabular}{|c|c|c|c|c|c|}
\hline Host & Tissue & $\begin{array}{l}\text { Samples } \\
(n)\end{array}$ & $\begin{array}{l}\text { Strains } \\
(n)\end{array}$ & Location & Year \\
\hline \multirow{7}{*}{$\begin{array}{l}\text { Maize (Zea } \\
\text { mays L.) }\end{array}$} & Seed & 10 & 11 & Xuzhou, Jiangsu & 2012 \\
\hline & Seed & 10 & 16 & Huaian, Jiangsu & 2012 \\
\hline & Seed & 10 & 12 & Suqian, Jiangsu & 2017 \\
\hline & Seed & 10 & 11 & Nantong, Jiangsu & 2017 \\
\hline & Seed & 10 & 16 & Yancheng, Jiangsu & 2017 \\
\hline & Seed & 10 & 12 & Huaian, Jiangsu & 2017 \\
\hline & Seed & 10 & 14 & Yangzhou, Jiangsu & 2017 \\
\hline \multirow{14}{*}{$\begin{array}{c}\text { Rice (Oryza } \\
\text { sativa } \mathrm{L} .)\end{array}$} & Stem & 4 & 16 & Yancheng, Jiangsu & 2012 \\
\hline & Stem & 4 & 13 & Huaian, Jiangsu & 2012 \\
\hline & Seed & 4 & 5 & Taizhou, Jiangsu & 2017 \\
\hline & Stem & 4 & 2 & & \\
\hline & Seed & 4 & 27 & Suqian, Jiangsu & 2017 \\
\hline & Stem & 4 & 3 & & \\
\hline & Seed & 4 & 12 & Huaian, Jiangsu & 2018 \\
\hline & Stem & 4 & 2 & & \\
\hline & Seed & 4 & 5 & Changzhou, Jiangsu & 2018 \\
\hline & Stem & 4 & 3 & & \\
\hline & Seed & 4 & 8 & Yancheng, Jiangsu & 2018 \\
\hline & Stem & 4 & 4 & & \\
\hline & Seed & 4 & 8 & Xuzhou, Jiangsu & 2018 \\
\hline & Stem & 4 & 8 & & \\
\hline \multirow{3}{*}{$\begin{array}{l}\text { Soybean } \\
\text { [Glycine max } \\
\text { (Linn.) Merr.] }\end{array}$} & Seed & 10 & 10 & Nantong, Jiangsu & 2017 \\
\hline & Seed & 10 & 9 & Yancheng, Jiangsu & 2017 \\
\hline & Seed & 10 & 10 & Huaian, Jiangsu & 2017 \\
\hline
\end{tabular}

\section{Materials and Methods}

Isolation of Fusarium species. Fungal strains used in this study were obtained from three hosts: namely, rice, maize, and soybean. A total of 32 rice plants with typical bakanae symptoms in stems were sampled in 2012 (eight samples), 2017 (eight samples), and 2018 (16 samples). In addition, approximately $1 \mathrm{~kg}$ of rice seeds from each field was collected at harvest in the latter 2 years. Seeds from a total of 70 freshly harvested maize plants $(1 \mathrm{~kg}$ each) were collected in 2012 and 2017, and 10 samples were collected from each location. For soybean, seeds from a total of 30 plants $(1 \mathrm{~kg}$ each) in three locations were sampled during the 2017 growing season.

From each sample, 50 symptomatic/asymptomatic seeds and 10 rice stems with symptoms were chosen randomly, surfacedsterilized with $1 \%$ sodium hypochlorite for $1 \mathrm{~min}$, rinsed twice in sterile distilled water, and placed in Petri dishes containing potato dextrose agar (PDA); the plates were incubated at $25^{\circ} \mathrm{C}$ for 3 days. Suspected Fusarium strains were transferred to carnation leaf agar plates for conidia production, and each strain was single-spored according to the Fusarium laboratory manual (Leslie and Summerell 2006). All strains were grown on PDA as the regular culture medium and stored in $20 \%$ glycerol solution at $-80^{\circ} \mathrm{C}$. Detailed information on all strains is listed in Table 1 .

Sequence analysis and phylogenetic reconstruction. Mycelia were harvested from the colonies of Fusarium species on PDA, and fungal DNA was extracted with the $2 \%$ cetyltrimethylammonium bromide (Leslie and Summerell 2006). Primers Fum1F1 (5'CACATCTGTGGGCGATCC-3')/Fum1R2 (5'-ATATGGCCCCAGC TGCATA- $3^{\prime}$ ) and EF1T (5'-ATGGGTAAGGAGGACAAGAC- $3^{\prime}$ )/ EF2T (5'-GGAAGTACCAGTGATCATGTT-3') were used for the amplification of $F U M 1$ and $T E F-1 \alpha$ gene fragments, respectively (O’Donnell et al. 1998a; Proctor et al. 2004, 2013). The primers were synthesized by an oligonucleotide synthesis facility (Shanghai Shenggong Biotechnological Ltd., Shanghai, China) and the PCRamplified DNA fragments were sequenced by the same company.

The phylogenetic relationships were reconstructed with the MEGA software package (Kumar et al. 2016) using the maximum parsimony approach. No gap-containing positions were considered in the phylogenetic analysis. The bootstrap method used a heuristic search with 1,000 replicates.

Pathogenicity tests. Rice germination test. A total of 144 strains representative of four species were selected for the evaluation of their abilities to affect seed germination. After surface sterilization, rice grains were soaked for $1 \mathrm{~h}$ in a $10^{6} / \mathrm{ml}$ spore suspension, placed on sterile water-moistened filter paper in Petri dishes, and then incubated at $25^{\circ} \mathrm{C}$ (three plates per strain $/ 10$ seeds per plate). The control seeds were soaked in sterile water. The number of germinated seeds was measured after incubation for 7 days.

Rice seedling test. Those strains were also tested for their pathogenicity in two conventional rice varieties (Nangeng 46 and Xinliangyou 6308) with the method described by Cruz et al. (2013). Each strain was grown in shaken potato dextrose broth and culture filtrates were passed through filter paper and a microporous filter. Surface-sterilized rice seeds were transferred to Petri dishes with sterile water-moistened filter paper and incubated at room temperature. Sprouting seeds and culture filtrates were added to a glass test tube and incubated at $25^{\circ} \mathrm{C}$ under alternating 12-h light/12-h darkness. At 14 days after incubation, elongation or stunting symptoms relative to the untreated control were evaluated by measuring the heights of seedlings.

Chemical analysis. The ability of the above 144 strains to produce mycotoxins was evaluated on autoclaved rice grains. Rice medium was prepared by weighing $30 \mathrm{~g}$ of rice grains into 100-ml Erlenmeyer flasks containing $10 \mathrm{ml}$ of distilled water. The flasks were autoclaved for $1 \mathrm{~h}$. Then, rice was stirred with a glass rod to separate rice kernels and the flasks were autoclaved again for $1 \mathrm{~h}$. Each strain was inoculated into the medium with 10 agar plugs of the PDA culture. After 20 days of incubation at $25^{\circ} \mathrm{C}$, the rice cultures were harvested, dried, and ground to a fine powder. Five grams of each rice culture was shaken with $20 \mathrm{ml}$ of acetonitrile/water $(1: 1, \mathrm{vol} / \mathrm{vol})$ treated with $0.1 \%$ methanoic acid (ROE Scientific Inc., Newark, DE). After centrifugation at 3,500 rpm 
for $5 \mathrm{~min}, 2 \mathrm{ml}$ of the supernatant was purified with an extraction salt pack (200 mg of $\mathrm{MgSO}_{4}, 100 \mathrm{mg}$ of sodium citrate, $100 \mathrm{mg}$ of $\mathrm{C}_{18}$, and $100 \mathrm{mg}$ of primary secondary amine). After centrifugation, the supernatant was transferred to a new glass tube. The extracts were concentrated under nitrogen, diluted with $0.6 \mathrm{ml}$ of extract solution, and filtered through a nylon filter ( $13 \mathrm{~mm}$ in diameter, $0.22 \mu \mathrm{m}$ pore size).

The toxins were analyzed with a LC-20ADXR liquid chromatograph (Shimadzu, Kyoto, Japan) coupled to an AB SCIEX Triple Quad mass spectrometer (Applied Biosystems, Foster City, CA). The analytical column was a Kinetex $100 \mathrm{~A} \mathrm{C}_{18}$ column $(100 \times$ $2.3 \mathrm{~mm}, 2.6 \mu \mathrm{m}$ ) from Phenomenex (Torrance, CA), and the column temperature was maintained at $40^{\circ} \mathrm{C}$. The flow rate was $0.5 \mathrm{ml} / \mathrm{min}$, and the injection volume was $2 \mu$. The mass spectrometric analyses were performed with the following operation parameters: gas temperature, $500^{\circ} \mathrm{C}$; gas flow rate, 10 liters $/ \mathrm{min}$; nebulizer gas pressure, $50 \mathrm{psi}$; and capillary voltage, $5,500 \mathrm{~V}$. Nitrogen was used as the ion source and the collision cell. Mycotoxins were analyzed via multiple reaction monitoring.

The mobile phase consisted of $5 \mathrm{mM}$ of ammonium acetate/ acetic acid (99.9/0.1, vol/vol) (A) and methanol (B). The separate high-performance liquid chromatography conditions were as follows: 0 to $0.01 \mathrm{~min}$, solvent A linearly increased to $10 \%$; 1.6 to 2 min, solvent A linearly increased from 10 to $35 \%$; 2 to $4.44 \mathrm{~min}$, solvent A linearly increased to $55 \%$; 4.44 to $6.5 \mathrm{~min}$, solvent A linearly increased from 55 to $90 \% ; 6.5$ to $12 \mathrm{~min}$, solvent $\mathrm{A}$ remained constant at $90 \%$; and 12 to $15 \mathrm{~min}$, solvent A remained constant at $10 \%$.

Quantitative reverse transcription PCR. Conidia $\left(10^{5} / \mathrm{ml}\right)$ of five FB-producing and five GA-producing strains were inoculated in liquid ICI media (containing $60 \mathrm{mM}$ of glutamine) and incubated for 3 days. Total RNA was extracted from mycelia with TRIzol reagent (Takara, Dalian, China). Reverse-transcribed complementary DNA was synthesized with the Prime-Script RT reagent kit (Takara). Real-time PCR was performed with SYBR Premix Ex Taq (Takara). All experimental procedures were performed according to the manufacturer's instructions. PCR assays were completed in a LightCycler 96 system (Roche, Basel, Switzerland), and the data were analyzed with sequence detector software. Gene expression profiles were normalized to $\beta$-tubulin gene expression, and relative changes in gene expression levels were calculated by the $\Delta \Delta$ cycle threshold method. Relative gene expression levels of $C P S / K S$ and $P 450$ in the GA biosynthetic pathway, FUM1 and FUM21 in the FB biosynthetic pathway, and several global regulators (AreA, AreB, Lae1, Vel, SGE1, and $C s m 1)$ of each strain were analyzed. The amplification levels of the genes in one GA-producing strain were used as a reference (set to 1.0). The primer sequences for these genes are listed in Supplementary Table S1.

Statistical analysis. Statistical comparisons of mycotoxin production and pathogenicity parameters between species were made with a $t$ test. All statistical analyses were performed with the SigmaStat statistical software package (SPSS, version 11). Differences with $P<$ 0.05 were considered significant.

\section{Results}

Phylogenetic analysis. Two hundred thirty-seven strains were recovered from the rice, maize, and soybean samples, and FFSC species were morphologically identified. Based on the $T E F-1 \alpha$ sequences submitted to the NCBI and Fusarium-ID databases, these strains were identified as $F$. verticillioides (68 strains), $F$. proliferatum (52 strains), F. fujikuroi (106 strains), and $F$. andiyazi (11 strains). The species composition varied among the host crops (Fig. 1). The maize samples were dominated by $F$. verticillioides $(56.5 \%)$ and $F$. proliferatum $(39.1 \%)$, whereas soybean samples were dominated by $F$. fujikuroi $(75.9 \%)$ and $F$. proliferatum $(20.7 \%)$. Four species were found in rice samples, including seeds and stems, and $F$. fujikuroi was dominant (69.0\%). Partial TEF-1 $\alpha$ sequences of approximately $600 \mathrm{bp}$ were amplified in all of the strains and the phylogenetic tree consisted of distinct clusters. One cluster was formed by the $F$. verticillioides strains, which were the most frequently detected in maize seeds, and $11 F$. andiyazi strains isolated from rice. The other cluster was composed of $F$. proliferatum, the second most common species associated with maize, and F. fujikuroi, mainly originating from rice seeds or stems. F. fujikuroi was divided into two groups: one consisting of 51 strains with low levels of FB production recovered from rice stem samples with only bakanae symptoms and the other group with stunting and a high FB production isolated from rice, maize, and soybean seeds.

The phylogenetic tree with the partial sequences of FUMlof the 133 strains showed three groups: $F$. verticillioides, $F$. proliferatum, and $F$. fujikuroi (Fig. 2). No amplification was detected in $F$. andiyazi strains.

The two trees were generally identical, as all of the strains except for the $11 F$. andiyazi strains were the same. Both figures illustrated no correlation with the sampling sites, years, and different hosts. In addition, $F$. verticillioides and $F$. andiyazi were phylogenetically closely related, while $F$. proliferatum and $F$. fujikuroi shared a close genetic relationship. All of the obtained TEF-1 $\alpha$ sequences were submitted to Genbank as accession numbers from MN695921 to MN696157.

Pathogenicity. The germination rate was significantly $(P<0.05)$ reduced after the incubation of rice seeds for 7 days with the FFSC strains compared with the control (Fig. 3A). The average percentages of germinated seeds were $76.8,73.3,75.6,79.3$, and $70.0 \%$ for $F$. verticillioides, $F$. proliferatum, $F$. fujikuroi-bakanae, $F$. fujikuroi, and $F$. andiyazi, respectively. There were no significant $(P<0.05)$ differences in the germination rate of rice seeds among the different species.

Either stunting or elongation was observed in rice seedlings inoculated with FFSC strains compared with the controls. F. fujikuroi strains from bakanae disease showed elongated seedlings that were typical bakanae symptoms in rice, whereas the FB-producing FFSC strains along with $F$. fujikuroi from other sources caused stunting symptoms in rice seedlings (Fig. 3B). The capacity to influence the development of rice seedlings did not differ between $F$. andiyazi and the untreated control.

Secondary metabolite synthesis. All 144 strains tested in the pathogenicity assay were analyzed for their ability to produce FBs (FB1, FB2, and FB3), hydrolyzed fumonisin B1 (HFB1), BEA, MON, and GA3 (Table 2). HFB1 was detected in 43 strains at low levels (5.1 to $44.7 \mu \mathrm{g} / \mathrm{kg}$ ); therefore, further analysis of HFB1 was not conducted.

FBs were the most abundant secondary metabolic products. In particular, FB1 was found in all $F$. verticillioides and $F$. proliferatum strains, ranging in production from 1,371.2 to $24,965.0$ and 99.6 to $16,355.5 \mu \mathrm{g} / \mathrm{kg}$, respectively. Similarly, the production of FB2 and FB3 in the whole $F$. verticillioides population ranged from 457.7 to $15,706.5$ and 97.1 to $4,840.9 \mu \mathrm{g} / \mathrm{kg}$, respectively. The production of FB2 and FB3 in all $F$. proliferatum strains ranged from 86.2 to $9,306.6$ and 30.2 to $2,885.0 \mu \mathrm{g} / \mathrm{kg}$, respectively. Two contrasting types of toxigenicity were associated with $F$. fujikuroi: 25 strains were associated with bakanae disease, among which 24 produced 5.8 to $141.9,6.2$ to 135.2 , and 6.2 to $310.4 \mu \mathrm{g} / \mathrm{kg}$ of FB1, FB2, and FB3, respectively; 37 strains did not cause bakanae disease, all of which synthesized $3,380.2$ to $17,496.0,2,057.2$ to $13,575.8$, and 146.6 to $2,763.6 \mu \mathrm{g} / \mathrm{kg}$ of FB1, FB2, and FB3, respectively. FB1, $\mathrm{FB} 2$, and FB3 were synthesized by $F$. andiyazi at low levels (ranging from 32.2 to $66.5,27.9$ to 48.8 , and 5.1 to $9.9 \mu \mathrm{g} / \mathrm{kg}$ for FB1, FB2, and FB3, respectively).

MON was produced in 27 of 33 strains of $F$. proliferatum $(4.0$ to $1,573.6 \mu \mathrm{g} / \mathrm{kg}), 21$ of 25 strains of $F$. fujikuroi-bakanae (1.5 to $1,688.4 \mu \mathrm{g} / \mathrm{kg}$ ), and 37 of 37 strains of $F$. fujikuroi (2.8 to $1,755.6 \mu \mathrm{g} / \mathrm{kg}$ ). Low detection rates and concentrations of MON were observed in $F$. verticillioides and $F$. andiyazi.

The capability of BEA synthesis was similar to that of MON synthesis, as BEA production by $F$. verticillioides and $F$. andiyazi was low compared with that by the other two Fusarium species. Twenty-eight of the $33 \mathrm{~F}$. proliferatum strains, 21 of the $25 \mathrm{~F}$. fujikuroi-bakanae strains, and 35 of the $37 \mathrm{~F}$. fujikuroi strains were BEA producers, with concentrations ranging from 4.0 to 1,573.6, 1.3 to $3,674.0$, and 7.4 to $2,507.4 \mu \mathrm{g} / \mathrm{kg}$, respectively. 
GA was found in only $F$. fujikuroi strains from rice stems with bakanae symptoms; the GA3 content ranged between 27.9 and $3,884.5 \mu \mathrm{g} / \mathrm{kg}$.

Molecular characterization of $\boldsymbol{F}$. fujikuroi. As the $F$. fujikuroi strains showed a markedly distinct cluster in sequence analysis of the TEF-1 $\alpha$ gene, the sequences of this marker from GAproducing and FB-producing $F$. fujikuroi strains were further analyzed. Multiple alignments of these sequences revealed common nucleotide substitutions at two sites; all FB producers had cytosine and thymine at nucleotide positions 447 and 618, respectively, and these two nucleotide sites contained adenine and guanine in FB nonproducers, respectively.

Considering the notable differences in the mycotoxin profiles between the two $F$. fujikuroi subgroups, we compared the expression patterns of two types of randomly selected strains with key GA ( CPS/KS and P450) and FB (FUM1 and FUM21) biosynthetic genes and several vital regulator genes (AreA, AreB, Lae1, Vel, SGE1, and $C s m 1)$. However, little change was observed in these regulatory genes between FB- and GA-producing F. fujikuroi strains (unpublished data), suggesting similar effects of these genes in the field strains. In contrast, the expression characteristics of the GA- and FB-synthetic genes differed dramatically. The FUM1 and FUM21 transcript levels of FB producers were significantly $(P<0.05)$ higher than those of GA producers. Furthermore, the bakanae-type strains showed higher expression levels of $C P S / K S$ and $P 450$ than the FBproducing strains (Fig. 4).

In addition, two other genes that encode unknown products (namely, PKS51 and NRPS31) were used to further distinguish the

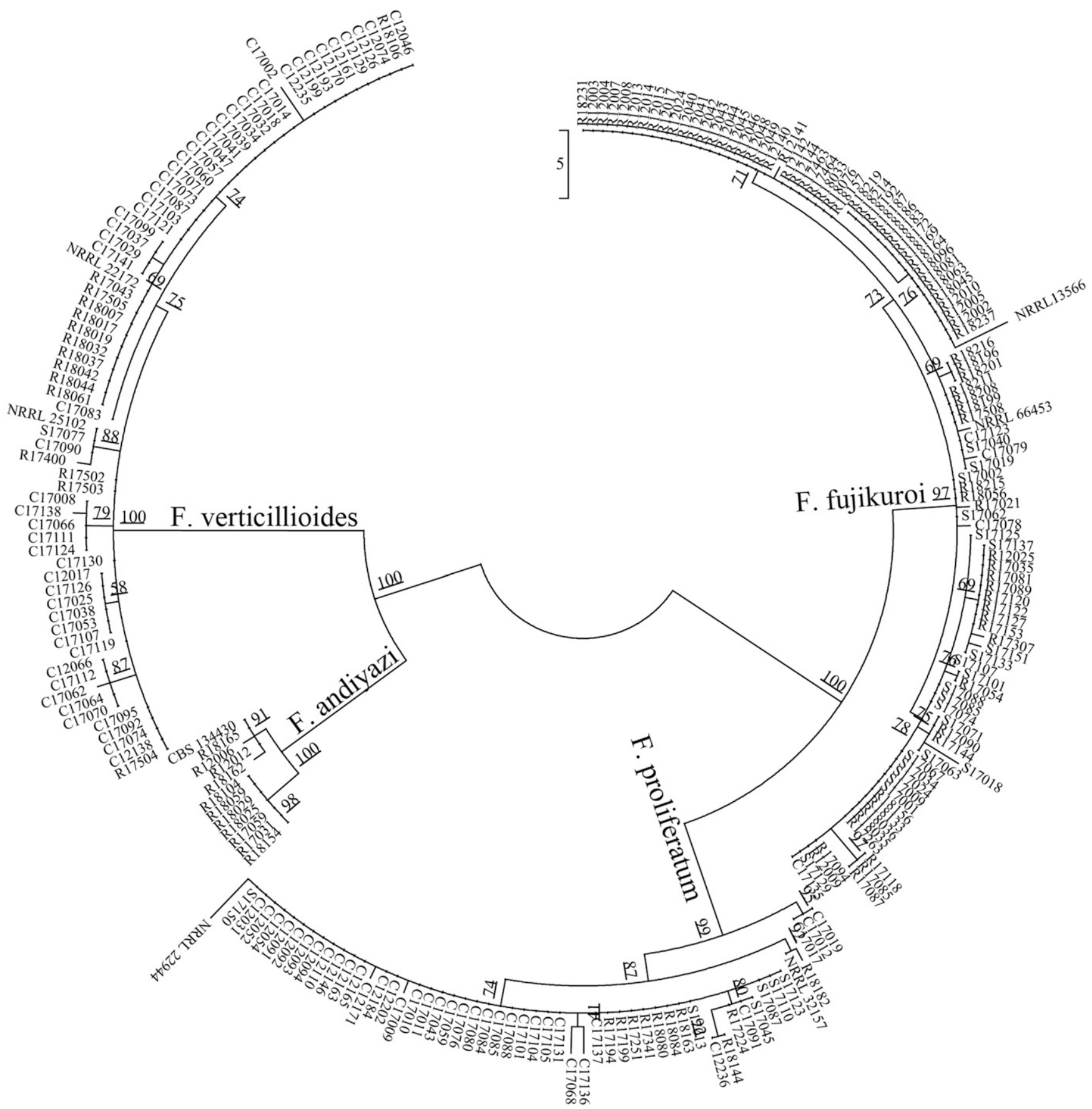

Fig. 1. Phylogenetic tree inferred from partial translation elongation factor $T E F-1 \alpha$ gene sequences from members of the Fusarium fujikuroi species complex was constructed by a maximum likelihood analysis. Reference sequences were from the latest literature. NRRL, Agricultural Research Service Culture Collection (USA); CBS, Centraalbureau voor Schimmelcultures (the Netherlands). Six NRRL strains (13566, 22172, 22944, 25102, 32157, and 66453) and one CBS strain (134430) were used as references. 
two groups. With the primer pair for $P K S 51$, only $F$. fujikuroi strains with high FB production successfully underwent PCR amplification of a 382-bp DNA fragment; however, an approximately 434-bp amplified product of NRPS31 was obtained from FB-nonproducing $F$. fujikuroi strains (Supplementary Fig. S1).

\section{Discussion}

The contamination of agricultural crops with Fusarium species belonging to the FFSC is a considerable public health hazard attributable to the production of deleterious secondary metabolites. Thus, there is an urgent need to identify the distribution pattern and toxin-producing ability of these Fusarium strains in diverse crops. Based on the reports in America (de Oliveira Rocha et al. 2011), Europe (Covarelli et al. 2012; Jurado et al. 2006), and Asia (Kim et al. 2012), F. verticillioides is the most predominant species in maize. In contrast, $F$. proliferatum has a wider host range and can colonize some nonstaple plants, such as date palm, giant reed, pineapple, garlic, and asparagus (Jurado et al. 2010; Waśkiewicz et al. 2013), in addition to wheat, maize, and rice (Jurado et al. 2006; Kim et al. 2012; Palacios et al. 2015). Combined with previous findings (Qiu et al. 2015), we found that $F$. verticillioides and $F$. proliferatum are the most common species in maize in our region. Our results further confirm that $F$. proliferatum can occur in soybean seeds at a low frequency. Although these species might not cause serious soybean disease, their impact on food safety cannot be ignored, as they can synthesize various toxic secondary metabolites.

We recovered $F$. fujikuroi strains from maize, soybean, and rice samples. It is well known that $F$. fujikuroi is the causal agent of the rice disease bakanae in the major rice-growing regions in the world

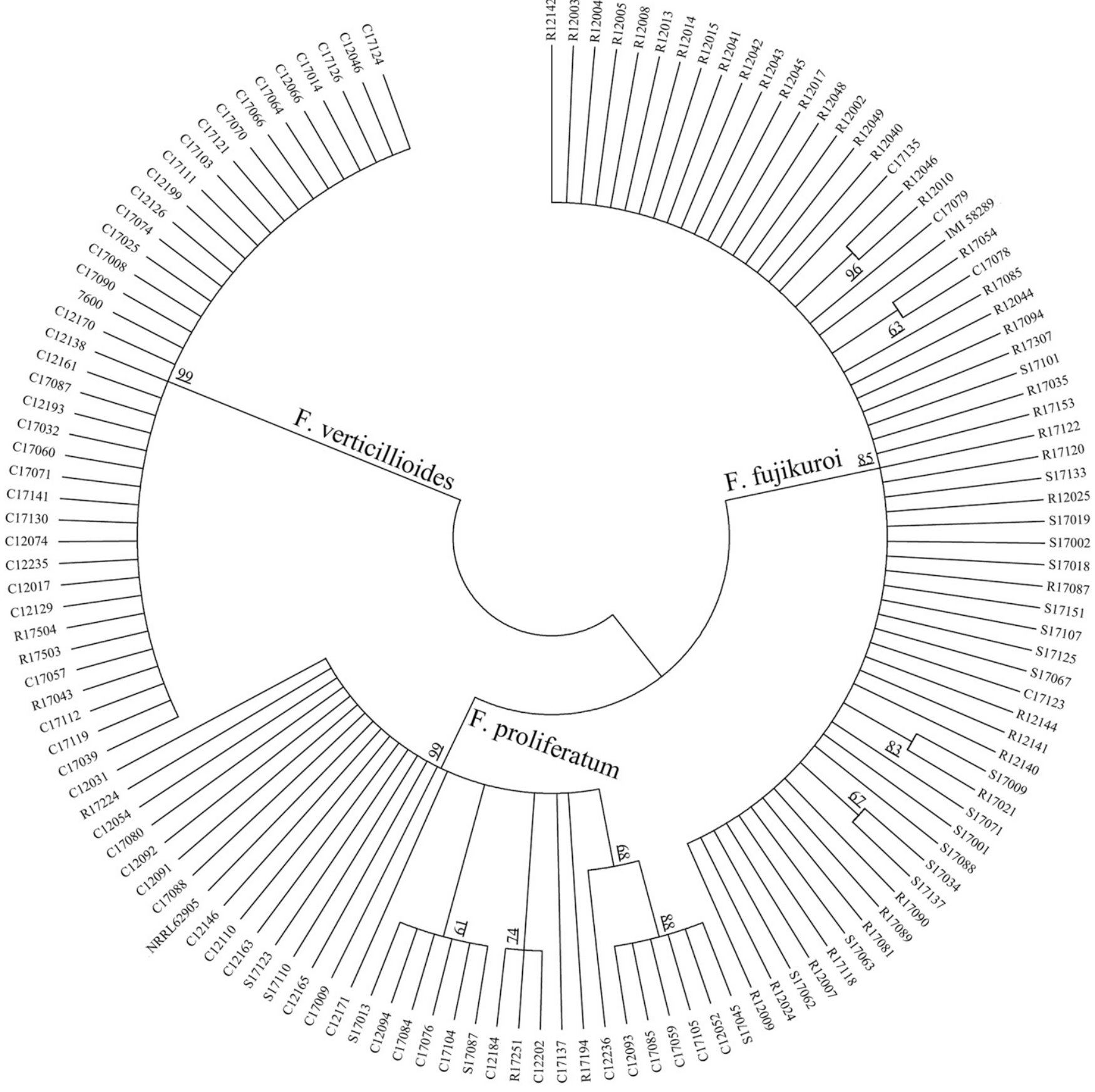

Fig. 2. Phylogenetic tree inferred from partial fumonisin synthetic gene FUM1 sequences of Fusarium fujikuroi species complex strains was constructed by a maximum likelihood analysis. Two sequenced standard strains, 7600 (F. verticillioides) and IMI 58289 (F. fujikuroi), along with NRRL 62905 were used as references. NRRL, Agricultural Research Service Culture Collection (USA). 
(Leslie and Summerell 2006). In our study, most of the F. fujikuroi strains were recovered from rice seeds or stems; however, we also recovered 4 and 17 strains of $F$. fujikuroi from maize and soybean seeds, respectively. Despite the prevalence, the $F$. fujikuroi strains we recovered were asymptomatic in the seeds of the three crops. Considering that the prevalent pathogen in soybean is $F$. solani (Jin et al. 1996) and that the occurrence of FFSC species on soybean is rare (Choi et al. 2018; Garcia et al. 2012), further investigation of the pathogenicity of $F$. fujikuroi on soybean is necessary. Moreover, a number of $F$. fujikuroi strains have been isolated from sugarcane and grapes (Bolton et al. 2016). We also detected 11 strains of $F$. andiyazi in rice samples; $F$. andiyazi was first described from sorghum (Marasas et al. 2001) and successively discovered on rice and maize from Africa and Asia (Hsuan et al. 2011; Wulff et al. 2010). To date, the host range of this pathogen is limited. This is the first report of $F$. andiyazi on rice in eastern China.

Fusarium species in the FFSC are difficult to distinguish by only morphological characteristics. In this study, the sequences of FUM1 and $T E F-1 \alpha$ were used for phylogenetic analysis, and the strains formed separate and well-supported groups. $F$. fujikuroi and $F$. proliferatum were grouped into one major clade, and $F$. andiyazi and $F$. verticillioides were closely related. However, Proctor et al. (2013) found inconsistent results regarding the evolution of the FFSC species based on 12 primary metabolism genes and nine FB biosynthetic genes. The authors suggested that the ancestor of the phylogenetic housekeeping genes was different from that of the biosynthetic genes. This discrepancy might result from the limited number of molecular markers used in our research.

Several findings showed similar results suggesting that $F$. fujikuroi and $F$. proliferatum are morphologically and biologically very similar and are located in the Asian clade or the neighboring group (Hsuan et al. 2011; O'Donnell et al. 1998b; Wulff et al. 2010). Although the detailed species concept and phylogenetic analysis of $F$. andiyazi remain to be determined (Kvas et al. 2009), the few relevant publications revealed that this species might be clustered in the main clade with $F$. verticillioides, an African clade member (Hsuan et al. 2011; Wulff et al. 2010). It was obvious that single species were separated into independent groups, and there was no correlation with geographic origin, host, or sampling year. The results of this and most previous studies (Covarelli et al. 2012; de Oliveira Rocha et al. 2011; Jurado et al. 2010) might be explained by the easy dispersal of the pathogens in nature, as they produce a large number of airborne conidia and have high adaptability to different habitats.

We confirmed the pathogenicity of FFSC strains using three different methods, of which the rice seedling test showed stunting or elongation of rice seedlings. Stunting and elongation of rice seedlings are typical symptoms of FFSC species and have been reported in several studies (Amoah et al. 1995; Cruz et al. 2013; Jeon et al. 2013; Wulff et al. 2010). Here, only $F$. fujikuroi-bakanae strains induced the growth of rice roots, and other species significantly $(P<$ 0.05 ) reduced the growth of the rice seedlings, leading to stunted seedlings. It was demonstrated that the elongation of rice seedlings was caused by GA production in $F$. fujikuroi (Bömke and Tudzynski 2009), and the ability of the fungus to synthesize GAs was favorable for efficient colonization in rice roots (Wiemann et al. 2013). F. verticillioides and $F$. proliferatum were associated with stunted growth of rice seedlings (Amoah et al. 1995; Desjardins et al. 2000) and were considered potential pathogens of rice (Jeon et al. 2013; Wulff et al. 2010). In a 2017 study, deletion of FUM1 and FUB1 (fusaric acid biosynthesis gene) in $F$. fujikuroi strain B14 caused stunting and the production of high amounts of FBs; fusaric acid, the lack of GA accumulation, and additional factors worked together and played crucial roles in causing stunted rice seedlings (Niehaus et al. 2017). The pathogenicity of FFSC strains depends on the balance of secondary metabolites, the environmental conditions, and the nutritional status of hosts (Amoah et al. 1995).
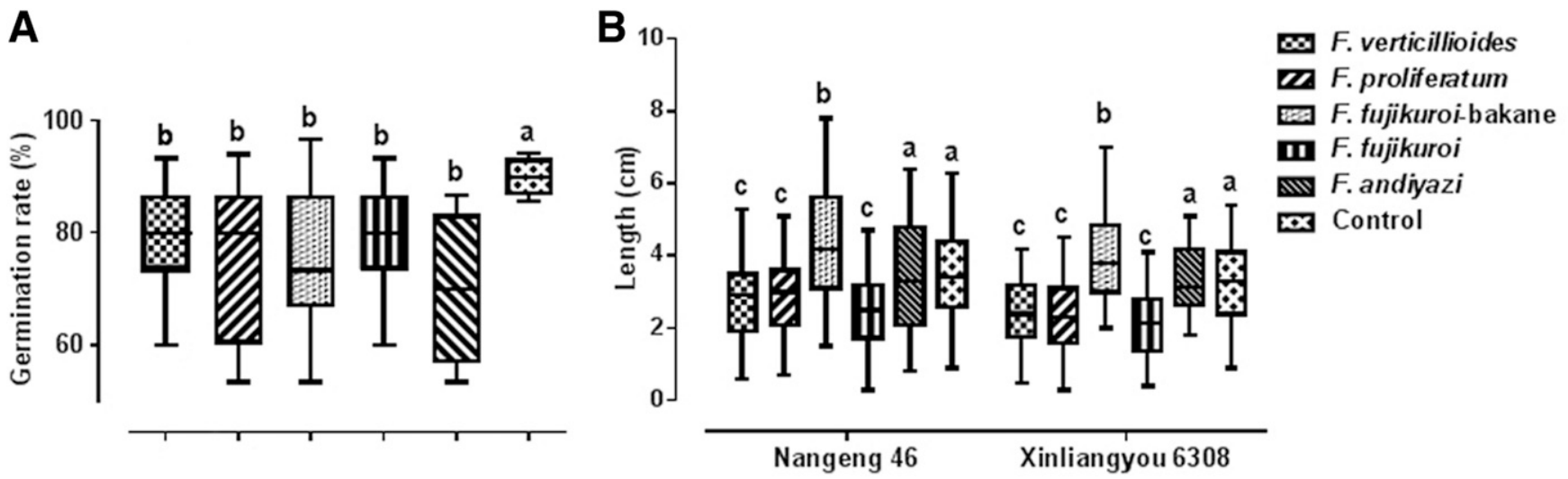

Fig. 3. Pathogenicity assay of 144 strains of the Fusarium fujikuroi species complex. A, The germination rate of the rice seeds after 7 days of spore treatment with each strain. B, Growth of the rice seedlings 7 days after inoculation with each strain. Different letters above bars indicate a significant difference at $P<0.05$ using a Tukey's student range test.

Table 2. Mycotoxin production of 144 strains of the Fusarium fujikuroi species complex

\begin{tabular}{|c|c|c|c|c|c|c|c|}
\hline \multirow[b]{2}{*}{ Species $(n)$} & \multicolumn{7}{|c|}{ Mycotoxins $(\mu \mathrm{g} / \mathrm{kg})^{\mathbf{a}}$} \\
\hline & FB1 & FB2 & FB3 & HFB1 & MON & BEA & GA3 \\
\hline $\begin{array}{l}\text { F. verticillioides } \\
\text { (38) }\end{array}$ & $\begin{array}{c}13,859.1 \pm 6,024.7 \\
(1,317.2-24,965.0)\end{array}$ & $\begin{array}{c}6,526.7 \pm 3,548.3 \\
(457.7-15,706.5)\end{array}$ & $\begin{array}{c}1,539.4 \pm 1,095.6 \\
(99.6-16,355.5)\end{array}$ & $\begin{array}{r}6.0 \pm 10.1 \\
(0-41.2)\end{array}$ & $\begin{array}{r}0.1 \pm 0.8 \\
(0-6.7)\end{array}$ & $\begin{array}{l}0.8 \pm 1.9 \\
(0-12.8)\end{array}$ & $<0$ \\
\hline $\begin{array}{l}\text { F. proliferatum } \\
\text { (33) }\end{array}$ & $\begin{array}{r}7,538.0 \pm 4,361.6 \\
(99.6-16,355.5)\end{array}$ & $\begin{array}{c}3,138.3 \pm 2,422.0 \\
(86.2-9,306.6)\end{array}$ & $\begin{array}{l}494.5 \pm 514.3 \\
\quad(30.2-2,885.0)\end{array}$ & $\begin{array}{l}2.17 \pm 4.5 \\
(5.8-15.7)\end{array}$ & $\begin{array}{c}115.5 \pm 275.0 \\
(4.0-1,573.6)\end{array}$ & $\begin{array}{c}622.9 \pm 537.5 \\
\quad(4.0-1,573.6)\end{array}$ & $<0$ \\
\hline $\begin{array}{l}\text { F. fujikuroi- } \\
\text { bakanae (25) }\end{array}$ & $\begin{array}{c}21.7 \pm 34.0 \\
(5.8-141.9)\end{array}$ & $\begin{array}{c}15.3 \pm 32.0 \\
(6.2-135.2)\end{array}$ & $\begin{array}{l}7.1 \pm 41.5 \\
(6.2-310.4)\end{array}$ & $<0$ & $\begin{array}{c}246.7 \pm 485.7 \\
(1.5-1,688.4)\end{array}$ & $\begin{array}{l}467.7 \pm 838.5 \\
\quad(1.3-3,674.0)\end{array}$ & $\begin{array}{r}1,062.7 \pm 1,023.8 \\
(27.9-3,884.5)\end{array}$ \\
\hline $\begin{array}{l}\text { F. fujikuroi } \\
\text { (37) }\end{array}$ & $\begin{array}{c}10,076.1 \pm 4,420.6 \\
(3,380.2-17,496.0)\end{array}$ & $\begin{array}{l}8,614.1 \pm 3,557.3 \\
(2,057.2-13,575.8)\end{array}$ & $\begin{array}{l}623.5 \pm 474.2 \\
(146.6-2,763.6)\end{array}$ & $\begin{array}{l}9.6 \pm 12.6 \\
(5.1-44.7)\end{array}$ & $\begin{array}{c}485.0 \pm 332.8 \\
(2.8-1,755.6)\end{array}$ & $\begin{array}{c}930.0 \pm 691.1 \\
(7.4-2,507.4)\end{array}$ & $<0$ \\
\hline $\begin{array}{l}\text { F. andiyazi } \\
\text { (11) }\end{array}$ & $\begin{array}{l}33.8 \pm 21.7 \\
(32.2-66.5)\end{array}$ & $\begin{array}{l}25.6 \pm 18.6 \\
(27.9-48.8)\end{array}$ & $\begin{array}{l}3.7 \pm 4.4 \\
(5.1-9.9)\end{array}$ & $<0$ & $\begin{array}{c}23.8 \pm 30.8 \\
(7.5-84.27)\end{array}$ & $\begin{array}{c}1.2 \pm 1.5 \\
(1.71-3.23)\end{array}$ & $<0$ \\
\hline
\end{tabular}

${ }^{\mathrm{a}} \mathrm{FB}$, fumonisin; HFB, hydrolyzed fumonisin; MON, moniliformin; BEA, beauvericin; GA, gibberellic acid. 
Mycotoxin production by the FFSC species was determined with rice culture. In the case of $\mathrm{FBs}$, the majority of the $F$. verticillioides and $F$. proliferatum strains produced high amounts of this type of toxic substance. $F$. proliferatum produced higher amounts of FBs than $F$. verticillioides in maize, soybean, and wheat substrates (Leslie et al. 2004; Palacios et al. 2015; Waśkiewicz et al. 2013). However, significant variability was not found in this study or in other relevant studies (Choi et al. 2018; de Oliveira Rocha et al. 2011). FB production by $F$. verticillioides and $F$. proliferatum might depend on the host source and culture conditions and may vary among different strains of the same species (Darnetty and Salleh 2013). In addition to the most common FBs, the emerging Fusarium toxins MON and BEA have been reported to be present in substantial quantities and represent potential health threats that need to be further investigated. The results obtained for $\mathrm{MON}$ and BEA production suggested that these two toxins are common metabolites of $F$.
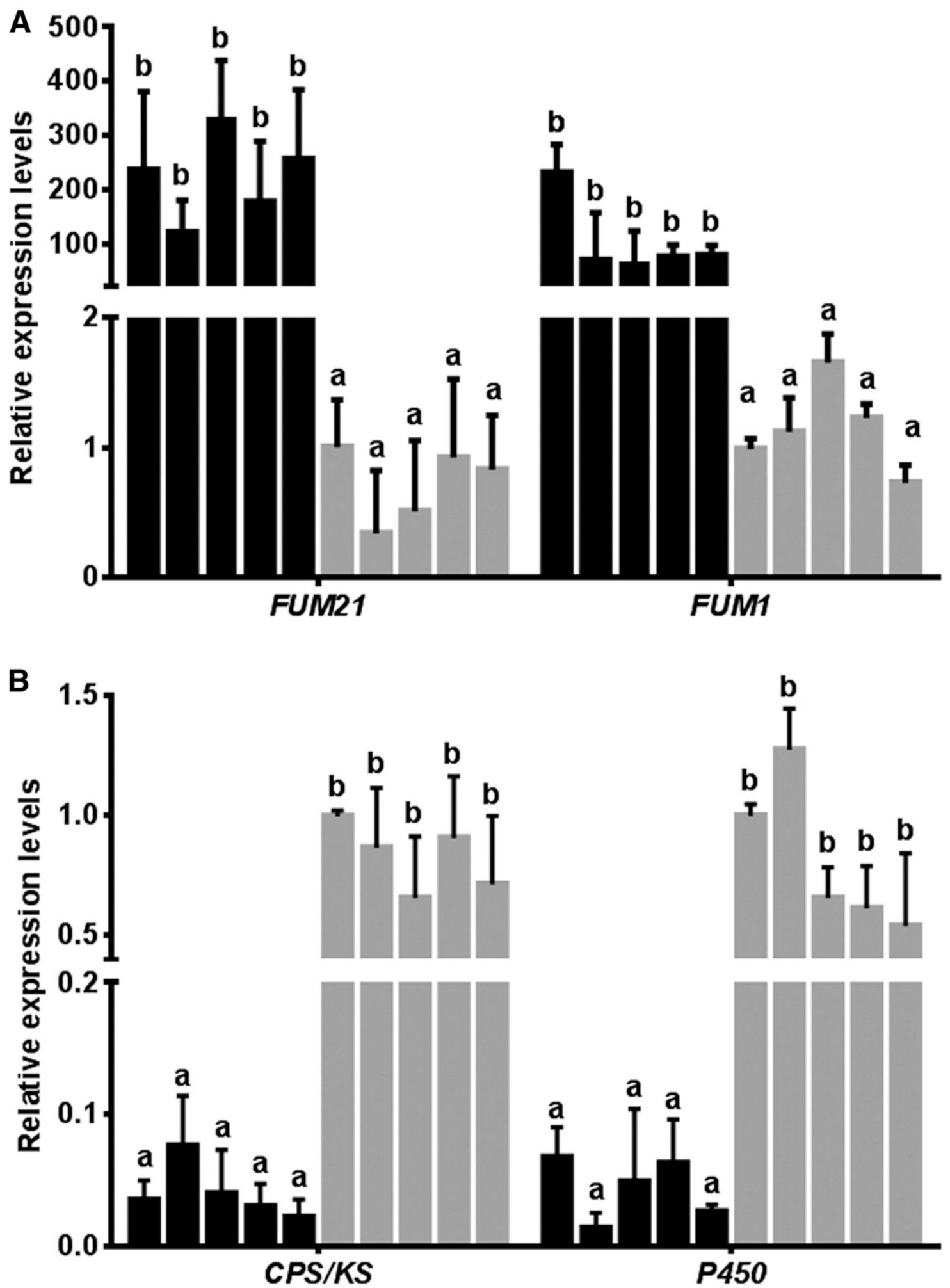

Fig. 4. Relative expression levels for the key genes involved in gibberellic acid and fumonisin biosynthesis of 10 strains of the Fusarium fujikuroi species complex. The transcript levels of A, FUM21 and FUM1 and B, CPS/KS and P450 were determined. Different letters above bars indicate a significant difference at $P<0.05$ using a Tukey's student range test. 
proliferatum and $F$. fujikuroi but not of other species. These findings are in accordance with several other reports (Leslie et al. 2004), and only a few strains could synthesize high amounts of both toxins.

GA and FB production seemed to act in opposition to each other in $F$. fujikuroi populations. F. fujikuroi-bakanae strains were able to produce GAs and low levels of FBs and cause typical bakanae symptoms. These results are consistent with previous studies on GA production by FFSC members (Desjardins et al. 2000; Wulff et al. 2010). In contrast, $F$. fujikuroi strains from nonbakanae rice seeds, maize, and soybean did not produce GA but produced high levels of FBs. Moreover, recent studies demonstrated that a number of $F$. fujikuroi strains from various sources produced a high amount of FBs (Bolton et al. 2016; Choi et al. 2018; Cruz et al. 2013; Suga et al. 2014, 2019). The defect of some genes in the FUM cluster was observed in some non-FB-producing strains of $F$. fujikuroi (Chiara et al. 2015; Suga et al. 2019), implying that a lack of essential genes for FB synthesis could result in a complete loss of FB production. However, the entire FB gene cluster was detected in most $F$. fujikuroi strains producing either no or only very small amounts of FBs (Niehaus et al. 2017), suggesting that other factors may also be involved in FBs production. Low levels of Fum 21 expression might be the cause of the low levels of FBs; the toxin yield increased 100-fold under the enhanced expression of this transcription factor (Rösler et al. 2016), suggesting that a $F$. fujikuroi strain with no FB synthesis can be transformed into a potent FB producer. However, an analysis of the effect of overexpression of Fum 21 on the transcription levels of other elements in the FUM cluster had not been performed. It is questionable whether Fum 21 is naturally silent. Several mutations in FUM21 and FUM7 were recently found, indicating causative variations leading to the loss of FB production (Sultana et al. 2019). In addition, single nucleotide polymorphisms (SNPs) have been reported in FUM8 and FUM18 (Proctor et al. 2013; Suga et al. 2019).

We compared the expression of biosynthetic genes of FBs (FUM1 and FUM21) and GAs (CPS/KS and P450) between F. fujikuroi strains and observed different expression characteristics. The results suggested that these genes might be used to differentiate the GA- and FB-producing $F$. fujikuroi strains. Some strains from the Philippines that were strongly capable of expressing FUM1 had undetectable levels of FBs, indicating the absence of FB production attributable to the lack of functionality of other synthetic genes (Cruz et al. 2013). In addition to these specific elements, variations in other sequences were observed in the two types of $F$. fujikuroi strains. TEF-1 $\alpha$ was suggested to contain a SNP (T618G) in Japanese $F$. fujikuroi groups (Suga et al. 2014). Here, another specific variation site (C447A) was identified. Although these mutations correlated with FB synthesis, SNPs were unlikely to be the cause of the variation in FB production because this molecular marker is a housekeeping gene. No connection between T618G and FBs synthesis was elucidated in F. fujikuroi from grapes (Bolton et al. 2016). These SNPs could be an effective tool in genetically characterizing GA and FB producers because of the long-term evolution and development of these subsequences.

In conclusion, we found a high degree of contamination of rice, maize, and soybean samples with different Fusarium species belonging to the FFSC and examined the pathogenicity and toxigenicity of the strains in this study. This contamination should be considered a potential public health threat with regard to mycotoxins produced at high levels by a large number of the strains.

\section{Literature Cited}

Abado-Becognee, K., Mobio, T. A., Ennamany, R., Fleurat-Lessard, F., Shier, W. T., Badria, F., and Creppy, E. E. 1998. Cytotoxicity of fumonisin B1: Implication of lipid peroxidation and inhibition of protein and DNA syntheses. Arch. Toxicol. 72:233-236.

Amoah, B. K., Rezanoor, H. N., Nicholson, P., and MacDonald, M. V. 1995. Variation in the Fusarium section Liseola: Pathogenicity and genetic studies of isolates of Fusarium moniliforme Sheldon from different hosts in Ghana. Plant Pathol. 44:563-572.

Bacon, C. W., Porter, J. K., Norred, W. P., and Leslie, J. F. 1996. Production of fusaric acid by Fusarium species. Appl. Environ. Microbiol. 62:4039-4043.

Baird, R., Abbas, H., Windham, G., Williams, P., Baird, S., Ma, P., Kelley, R., Hawkins, L., and Scruggs, M. 2008. Identification of select fumonisin forming Fusarium species using PCR applications of the polyketide synthase gene and its relationship to fumonisin production in vitro. Int. J. Mol. Sci. 9: 554-570.

Bolton, S., Brannen, P., and Glenn, A. 2016. A novel population of Fusarium fujikuroi isolated from Southeastern US winegrapes reveals the need to reevaluate the species' fumonisin production. Toxins (Basel) 8:254.

Bömke, C., and Tudzynski, B. 2009. Diversity, regulation, and evolution of the gibberellin biosynthetic pathway in fungi compared to plants and bacteria Phytochemistry 70:1876-1893.

Cerdá-Olmedo, E., Fernández-Martín, R., and Ávalos, J. 1994. Genetics and gibberellin production in Gibberella fujikuroi. Anton. Leeuw. Int. J. G. 65: 217-225.

Chiara, M., Fanelli, F., Mule, G., Logrieco, A. F., Pesole, G., Leslie, J. F., Horner, D. S., and Toomajian, C. 2015. Genome sequencing of multiple isolates highlights subtelomeric genomic diversity within Fusarium fujikuroi. Genome Biol. Evol. 7:3062-3069.

Choi, J. H., Lee, S., Nah, J. Y., Kim, H. K., Paek, J. S., Lee, S., Ham, H., Hong S. H., Yun, S. H., and Lee, T. 2018. Species composition of and fumonisin production by the Fusarium fujikuroi species complex isolated from Korean cereals. Int. J. Food Microbiol. 267:62-69.

Clemens, S., and Ma, J. F. 2016. Toxic heavy metal and metalloid accumulation in crop plants and foods. Annu. Rev. Plant Biol. 67:489-512.

Covarelli, L., Stifano, S., Beccari, G., Raggi, L., Lattanzio, V. M. T., and Albertini, E. 2012. Characterization of Fusarium verticillioides strains isolated from maize in Italy: Fumonisin production, pathogenicity and genetic variability. Food Microbiol. 31:17-24.

Cruz, A., Marín, P., González-Jaén, M. T., Aguilar, K. G. I., and Cumagun, C. J. R. 2013. Phylogenetic analysis, fumonisin production and pathogenicity of Fusarium fujikuroi strains isolated from rice in the Philippines. J. Sci. Food Agric. 93:3032-3039.

Darnetty, T., and Salleh, B. 2013. Toxigenicity of Fusarium species in Gibberella fujikuroi species complex (GFSC) associated with stalk and ear rot disease of corn. eSci J. Plant Pathol. 2:147-154.

de Oliveira Rocha, L., Reis, G. M., Da Silva, V. N., Braghini, R., Teixeira, M. M. G., and Corrêa, B. 2011. Molecular characterization and fumonisin production by Fusarium verticillioides isolated from corn grains of different geographic origins in Brazil. Int. J. Food Microbiol. 145:9-21.

Desjardins, A. E., Manandhar, H. K., Plattner, R. D., Manandhar, G. G., Poling, S. M., and Maragos, C. M. 2000. Fusarium species from Nepalese rice and production of mycotoxins and gibberellic acid by selected species. Appl. Environ. Microbiol. 66:1020-1025.

Ferrigo, D., Raiola, A., and Causin, R. 2016. Fusarium toxins in cereals: Occurrence, legislation, factors promoting the appearance and their management. Molecules 21:627.

Garcia, D., Barros, G., Chulze, S., Ramos, A. J., Sanchis, V., and Marín, S. 2012 Impact of cycling temperatures on Fusarium verticillioides and Fusarium graminearum growth and mycotoxins production in soybean. J. Sci. Food Agric. 92:2952-2959.

Gelderblom, W. C., Abel, S., Smuts, C. M., Marnewick, J., Marasas, W. F., Lemmer, E. R., and Ramljak, D. 2001. Fumonisin-induced hepatocarcinogenesis: Mechanisms related to cancer initiation and promotion. Environ. Health Perspect. 109:291-300.

Grewal, A. S., Grewal, A. S., Singla, A., Kamboj, P., and Dua, J. S. 2017. Pesticide residues in food grains, vegetables and fruits: A hazard to human health. J. Med. Chem. Toxicol. 2:40-46.

Hsuan, H. M., Salleh, B., and Zakaria, L. 2011. Molecular identification of Fusarium species in Gibberella fujikuroi species complex from rice, sugarcane and maize from Peninsular Malaysia. Int. J. Mol. Sci. 12:6722-6732.

Jeon, Y. A., Yu, S. H., Lee, Y. Y., Park, H. J., Lee, S., Sung, J. S., Kim, Y. G., and Lee, H. S. 2013. Incidence, molecular characteristics and pathogenicity of Gibberella fujikuroi species complex associated with rice seeds from Asian countries. Mycobiology 41:225-233.

Jin, H., Hartman, G. L., Nickell, C. D., and Widholm, J. M. 1996. Characterization and purification of a phytotoxin produced by Fusarium solani, the causal agent of soybean sudden death syndrome. Phytopathology 86:277-282.

Jurado, M., Marín, P., Callejas, C., Moretti, A., Vázquez, C., and González-Jaén, M. T. 2010. Genetic variability and fumonisin production by Fusarium proliferatum. Food Microbiol. 27:50-57.

Jurado, M., Vázquez, C., Callejas, C., and González-Jaén, M. T. 2006. Occurrence and variability of mycotoxigenic Fusarium species associated to wheat and maize in the South West of Spain. Mycotoxin Res. 22:87-91.

Kim, J. H., Kang, M. R., Kim, H. K., Lee, S. H., Lee, T., and Yun, S. H. 2012. Population structure of the Gibberella fujikuroi species complex associated with rice and corn in Korea. Plant Pathol. J. 28:357-363.

Kumar, S., Stecher, G., and Tamura, K. 2016. MEGA7: Molecular evolutionary genetics analysis version 7.0 for bigger datasets. Mol. Biol. Evol. 33: $1870-1874$.

Kvas, M., Marasas, W. F. O., Wingfield, B. D., Wingfield, M. J., and Steenkamp, E. T. 2009. Diversity and evolution of Fusarium species in the Gibberella fujikuroi complex. Fungal Divers. 34:1-21.

Lee, H. J., and Ryu, D. 2017. Worldwide occurrence of mycotoxins in cereals and cereal-derived food products: Public health perspectives of their co-occurrence. J. Agric. Food Chem. 65:7034-7051. 
Leslie, J. F. 1995. Gibberella fujikuroi: Available populations and variable traits. Can. J. Bot. 73 (Suppl 1):282-291.

Leslie, J. F., and Summerell, B. A., eds. 2006. The Fusarium Laboratory Manual. Blackwell Publishing, Oxford, UK.

Leslie, J. F., Zeller, K. A., Logrieco, A., Mule, G., Moretti, A., and Ritieni, A. 2004. Species diversity of and toxin production by Gibberella fujikuroi species complex strains isolated from native prairie grasses in Kansas. Appl. Environ. Microbiol. 70:2254-2262.

Logrieco, A., Moretti, A., Castella, G., Kostecki, M., Golinski, P., Ritieni, A., and Chelkowski, J. 1998. Beauvericin production by Fusarium species. Appl. Environ. Microbiol. 64:3084-3088.

Marasas, W. F., Rheeder, J. P., Lamprecht, S. C., Zeller, K. A., and Leslie, J. F. 2001. Fusarium andiyazi sp. nov., a new species from sorghum. Mycologia 93:1203-1210.

Marasas, W. F. O., Thiel, P. G., Rabie, C. J., Nelson, P. E., and Toussoun, T. A. 1986. Moniliformin production in Fusarium section Liseola. Mycologia 78:242-247.

Moretti, A. A., Logrieco, A., Bottalico, A., Ritieni, A., Fogliano, V., and Randazzo, G. 1996. Diversity in beauvericin and fusaproliferin production by different populations of Gibberella fujikuroi (Fusarium section Liseola). Sydowia 48:44-56.

Nicholson, P., Simpson, D. R., Wilson, A. H., Chandler, E., and Thomsett, M. 2004. Detection and differentiation of trichothecene and enniatin-producing Fusarium species on small-grain cereals. Eur. J. Plant Pathol. 110:503-514.

Niehaus, E. M., Kim, H. K., Münsterkötter, M., Janevska, S., Arndt, B., Kalinina, S. A., Houterman, P. M., Ahn, I. P., Alberti, I., Tonti, S., Kim, D. W., Sieber, C. M. K., Humpf, H. U., Yun, S. H., Güldener, U., and Tudzynski, B. 2017. Comparative genomics of geographically distant Fusarium fujikuroi isolates revealed two distinct pathotypes correlating with secondary metabolite profiles. PLoS Pathog 13:e1006670.

O’Donnell, K., Cigelnik, E., and Nirenberg, H. I. 1998b. Molecular systematics and phylogeography of the Gibberella fujikuroi species complex. Mycologia 90:465-493.

O’Donnell, K., Kistler, H. C., Cigelnik, E., and Ploetz, R. C. 1998a. Multiple evolutionary origins of the fungus causing Panama disease of banana: Concordant evidence from nuclear and mitochondrial gene genealogies. Proc. Natl. Acad. Sci. USA 95:2044-2049.

O’Donnell, K., Nirenberg, H. I., Aoki, T., and Cigelnik, E. 2000. A multigene phylogeny of the Gibberella fujikuroi species complex: Detection of additional phylogenetically distinct species. Mycoscience 41:61-78.

O'Donnell, K., Ward, T. J., Robert, V. A., Crous, P. W., Geiser, D. M., and Kang, S. 2015. DNA sequence-based identification of Fusarium: Current status and future directions. Phytoparasitica 43:583-595.

Palacios, S. A., Susca, A., Haidukowski, M., Stea, G., Cendoya, E., Ramirez, M. L., Chulze, S. N., Farnochi, M. C., Moretti, A., and Torres, A. M. 2015. Genetic variability and fumonisin production by Fusarium proliferatum isolated from durum wheat grains in Argentina. Int. J. Food Microbiol. 201: 35-41.

Proctor, R. H., Desjardins, A. E., Plattner, R. D., and Hohn, T. M. 1999. A polyketide synthase gene required for biosynthesis of fumonisin mycotoxins in Gibberella fujikuroi mating population A. Fungal Genet. Biol. 27:100-112.

Proctor, R. H., Plattner, R. D., Brown, D. W., Seo, J. A., and Lee, Y. W. 2004. Discontinuous distribution of fumonisin biosynthetic genes in the Gibberella fujikuroi species complex. Mycol. Res. 108:815-822.

Proctor, R. H., Van Hove, F., Susca, A., Stea, G., Busman, M., van der Lee, T., Waalwijk, C., Moretti, A., and Ward, T. J. 2013. Birth, death and horizontal transfer of the fumonisin biosynthetic gene cluster during the evolutionary diversification of Fusarium. Mol. Microbiol. 90:290-306.

Qiu, J., Xu, J., Dong, F., Yin, X., and Shi, J. 2015. Isolation and characterization of Fusarium verticillioides from maize in eastern China. Eur. J. Plant Pathol. 142: 791-800.

Rheeder, J. P., Marasas, W. F., and Vismer, H. F. 2002. Production of fumonisin analogs by Fusarium species. Appl. Environ. Microbiol. 68:2101-2105.

Rösler, S. M., Sieber, C. M., Humpf, H. U., and Tudzynski, B. 2016. Interplay between pathway-specific and global regulation of the fumonisin gene cluster in the rice pathogen Fusarium fujikuroi. Appl. Microbiol. Biotechnol. 100: $5869-5882$.

Stępień, Ł. 2014. The use of Fusarium secondary metabolite biosynthetic genes in chemotypic and phylogenetic studies. Crit. Rev. Microbiol. 40:176-185.

Stockmann-Juvala, H., and Savolainen, K. 2008. A review of the toxic effects and mechanisms of action of fumonisin B1. Hum. Exp. Toxicol. 27:799-809.

Suga, H., Arai, M., Fukasawa, E., Motohashi, K., Nakagawa, H., Tateishi, H., Fuji, S. I., Shimizu, M., Kageyama, K., and Hyakumachi, M. 2019. Genetic differentiation associated with fumonisin and gibberellin production in Japanese Fusarium fujikuroi. Appl. Environ. Microbiol. 85:e02414-e02418.

Suga, H., Kitajima, M., Nagumo, R., Tsukiboshi, T., Uegaki, R., Nakajima, T., Kushiro, M., Nakagawa, H., Shimizu, M., Kageyama, K., and Hyakumachi, M. 2014. A single nucleotide polymorphism in the translation elongation factor $1 \alpha$ gene correlates with the ability to produce fumonisin in Japanese Fusarium fujikuroi. Fungal Biol-UK 118:402-412.

Sultana, S., Kitajima, M., Kobayashi, H., Nakagawa, H., Shimizu, M., Kageyama, K., and Suga, H. 2019. A natural variation of fumonisin gene cluster associated with fumonisin production difference in Fusarium fujikuroi. Toxins (Basel) 11: 200

Waśkiewicz, A., Stępień, Ł., Wilman, K., and Kachlicki, P. 2013. Diversity of peaassociated $F$. proliferatum and $F$. verticillioides populations revealed by FUMI sequence analysis and fumonisin biosynthesis. Toxins (Basel) 5:488-503.

Wiemann, P., Sieber, C. M., Von Bargen, K. W., Studt, L., Niehaus, E. M., Espino, J. J., Huß, K., Michielse, C. B., Albermann, S., Wagner, D., Bergner, S. V., Connolly, L. R., Fischer, A., Reuter, G., Kleigrewe, K., Bald, T., Wingfield, B. D., Ophir, R., Freeman, S., Hippler, M., Smith, K. M., Brown, D. W., Proctor, R. H., Munsterkotter, M., Freitag, M., Humpf, H., Guldener, U., and Tudzynski, B. 2013. Deciphering the cryptic genome: Genome-wide analyses of the rice pathogen Fusarium fujikuroi reveal complex regulation of secondary metabolism and novel metabolites. PLoS Pathog 9:e1003475.

Wulff, E. G., Sørensen, J. L., Lübeck, M., Nielsen, K. F., Thrane, U., and Torp, J. 2010. Fusarium spp. associated with rice bakanae: Ecology, genetic diversity, pathogenicity and toxigenicity. Environ. Microbiol. 12:649-657. 